\section{Adaptação da versão saúde bucal do instrumento de diagnóstico do estágio de desenvolvimento da rede de atenção à saúde}

\author{
Adaptation of the oral health version of an \\ instrument for diagnosing the healthcare network's \\ stage of development
}

\author{
Análisis de medidas de adaptación de una \\ herramienta de diagnóstico de salud bucal para \\ evaluar una etapa de desarrollo en la atención \\ de la salud
}

Daniele Lopes Leal 1 Saul Martins Paiva ${ }^{1}$ Marcos Azeredo Furquim Werneck 1 Ana Cristina Borges de Oliveira ${ }^{1}$

\author{
1 Faculdade de Odontologia, \\ Universidade Federal de \\ Minas Gerais, Belo Horizonte, \\ Brasil. \\ Correspondência \\ A. C. B. Oliveira \\ Faculdade de Odontologia, \\ Universidade Federal de \\ Minas Gerais. \\ Av. Antônio Carlos 6627, \\ Belo Horizonte, $M G$ \\ 31270-901, Brasil. \\ anacboliveira@yahoo.com.br
}

\begin{abstract}
The implementation of oral healthcare networks is a recent process that requires evaluation. The current study aimed to describe the stages in the adaptation of the oral healthcare version of an instrument to evaluate the stage of development in the healthcare network under the Unified National Health System (SUS). The original instrument, called Instrument for the Diagnosis of Developmental Stages in the Healthcare Network, was developed by Mendes (2011). A formal evaluation was made of the general and referential meanings from the original instrument, producing the adapted version. The instrument was analyzed by a committee of oral health experts. Comprehension of the adapted version was evaluated using a pretest applied to 20 municipal oral health coordinators in Minas Gerais State. The results showed good conceptual, functional, item, semantic, and operational equivalence between the instruments. The oral health version was well understood and proved adequate for testing its psychometric properties. The latter stage is under completion.
\end{abstract}

Validation Studies; Questionnaires; Oral Health

\section{Resumo}

A implantação de redes de atenção à saúde bucal é um processo recente e necessita de ser avaliado. Este estudo objetivou descrever as etapas de adaptação da versão saúde bucal de um instrumento para avaliar o estágio de desenvolvimento da rede de atenção à saúde no Sistema Único de Saúde do Brasil. O instrumento original, denominado Instrumento de Diagnóstico do Estágio de Desenvolvimento da Rede de Atenção à Saúde, foi desenvolvido por Mendes (2011). Foi realizada a avaliação formal dos significados geral $e$ referencial do instrumento original, chegandose à versão adaptada. Para isso, o instrumento foi analisado por um comitê de especialistas da área em questão. A compreensão da versão adaptada foi avaliada em um pré-teste aplicado para $20 \mathrm{co}$ ordenadores municipais de saúde bucal de Minas Gerais. Constatou-se uma boa equivalência conceitual, funcional, de itens, semântica e operacional entre os instrumentos. A versão saúde bucal foi bem compreendida, apresentando-se adequada para ser testada quanto às suas propriedades psicométricas. Essa etapa se encontra em fase de conclusão.

Estudos de Validação; Questionários; Saúde Bucal 


\section{Introdução}

Há necessidade de se conhecer as respostas dadas pelos serviços às condições agudas e crônicas, apontando as estratégias para a organização do cuidado sob a responsabilidade de um serviço em rede 1,2,3,4,5,6. “...As RAS [redes de atenção à saúde] são organizações poliárquicas de conjuntos de serviços de saúde, vinculados entre si por uma missão única, por objetivos comuns e por uma ação cooperativa e interdependente, que permitem ofertar uma atenção contínua e integral à determinada população, coordenada pela atenção primária à saúde” 4 (p. 82).

Ao se buscar na literatura um instrumento capaz de investigar a avaliação do desenvolvimento da rede de atenção à saúde bucal, identificou-se um que analisa o estágio de desenvolvimento das redes de atenção à saúde: Instrumento de Diagnóstico do Estágio de Desenvolvimento da Rede de Atenção à Saúde 4 . Apresenta 172 questões em sete domínios: população, atenção primária à saúde, pontos de atenção à saúde secundária e terciária, sistemas de apoio, sistemas logísticos, sistema de governança da rede e modelo de atenção à saúde. Não aborda, no entanto, especificidades de saúde bucal.

O instrumento não sofreu validação teórica formal. Foi utilizado pela Secretaria de Estado de Saúde de Minas Gerais (SES/MG) durante a Oficina 1 do Plano Diretor de Atenção Primária à Saúde (PDAPS). O PDAPS é uma proposta educacional para capacitar profissionais em estratégias da gestão de processos de trabalho da atenção primária.

Este estudo objetivou descrever as etapas de adaptação da versão saúde bucal do instrumento de diagnóstico do estágio de desenvolvimento da rede de atenção à saúde.

\section{Metodologia}

A adaptação do instrumento seguiu a metodologia de Herdman et al. 7,8 e de Reichenheim \& Moraes 9 . Foram cumpridas as etapas de avaliação das equivalências conceitual e de item (pré-teste) e a validação de face 7,8,9,10,11. O instrumento é direcionado aos coordenadores de saúde bucal, municipais e estaduais. Tendo em vista o processo de validação do instrumento, o mesmo foi realizado e será descrito em outro artigo.

A versão saúde bucal foi analisada por uma equipe de quatro estudiosos. Foram discutidos pontos teóricos e conceituais sobre os dois instrumentos para verificar a equivalência conceitual e funcional entre eles e a validação de face. Esse momento permitiu avaliar se os domínios e questões eram importantes. A equivalência operacional de itens e a mensuração do instrumento foram realizadas na fase pré-teste 10,11 .

O pré-teste foi feito com vinte coordenadores municipais de saúde bucal de Minas Gerais. Foram convidados por telefone/e-mail, obtidos com as Superintendências/Gerências Regionais de Saúde da SES/MG. Os critérios de inclusão da amostra foram: ser cirurgião-dentista e exercer o cargo de coordenador municipal de saúde bucal.

Os coordenadores receberam (via correio) o termo de consentimento, o instrumento versão saúde bucal e um formulário sobre as características individuais (idade, sexo, tempo de formação em odontologia, escolaridade, tempo que exerce o cargo de coordenador e vínculo empregatício). Os participantes reenviaram o material via correio.

O estudo foi aprovado pelo Comitê de Ética em Pesquisa da Universidade Federal de Minas Gerais.

\section{Resultados}

Os quatro estudiosos que analisaram a versão original concordaram com o fato de que os domínios se relacionam às especificidades da rede de atenção à saúde bucal. Concordaram sobre a relevância e aplicabilidade dos itens do instrumento original e propuseram alterações em alguns itens que não eram específicos à saúde bucal. As modificações foram realizadas após consenso entre os pesquisadores. Para a validação de face, os quatro estudiosos concordaram com a construção proposta conforme a evidência teórica formulada para o novo instrumento.

A análise da versão saúde bucal resultou, para significado referencial, $17,6 \%$ de itens com modificações importantes. Houve ajustes na sintaxe de algumas frases com base na avaliação da versão saúde bucal. Para o significado conotativo, $70 \%$ dos itens tiveram significados inalterados, $15 \%$ pouco alterado, 7,5\% muito alterado e 7,5\% completamente alterado.

O instrumento adaptado foi mantido com os domínios do original (Figura 1). No domínio "população", foram adaptadas 11 questões e excluídas três do original. No domínio "atenção primária à saúde”, foram mantidas cinco questões, quatro foram adaptadas, oito foram excluídas e seis foram acrescentadas. No domínio "os pontos de atenção à saúde secundários e terciários”, foram excluídas todas as questões do instrumento original e acrescentadas 19 questões. No domínio "os sistemas de apoio", foram excluídas todas as questões do original e acrescentadas 15. Foram mantidas duas questões no domínio "os sistemas 
Figura 1

Avaliação da equivalência entre o Instrumento de Diagnóstico do Estágio de Desenvolvimento da Rede de Atenção à Saúde e a versão saúde bucal.

\section{Domínio: POPULAÇÃO}

\section{Questões adaptadas do instrumento original}

1. Há uma população claramente definida sob a responsabilidade da rede de atenção em saúde bucal?

2. Há uma clara definição das necessidades de saúde bucal desta população?

3. A estrutura operacional da rede de saúde bucal é formatada em função dessas necessidades?

4. Há um processo de territorialização da rede de saúde bucal?

5. A rede de saúde bucal segue um Plano Diretor de Regionalização?

6. Estão identificados os territórios sanitários com escala adequada para a oferta dos pontos de atenção à saúde bucal secundários?

7. Estão identificados os territórios sanitários com escala adequada para a oferta dos pontos de atenção à saúde bucal terciários?

8. Toda a população de responsabilidade da rede de saúde bucal está cadastrada, por unidade familiar, na APS?

9. As unidades familiares estão classificadas (de acordo com a vulnerabilidade social) por risco social?

10. A população está subdividida por grupos de idade (perfil demográfico)?

11. A população está subdividida por fatores de risco biológico?

Questões excluídas do instrumento original

$6,9,14$

Domínio: ATENÇÃO PRIMÁRIA À SAÚDE (APS)

Questões mantidas do instrumento original

12. A equipe da atenção primária à saúde tem claro o seu papel de responsabilização por sua população adscrita?

21. O atendimento na atenção primária é um requisito para o acesso aos outros níveis de atenção à saúde?

23. O prontuário utilizado na atenção primária à saúde está estruturado por família?

24. Há uma boa interação entre as equipes de atenção primária à saúde e de atenção especializada (consultas ou orientações rotineiras por escrito, por correio eletrônico ou sessões clínicas conjuntas)?

26. Existe um Conselho Local de Saúde eficaz no controle social da atenção primária à saúde?

Questões adaptadas do instrumento original

17. São utilizados protocolos clínicos relativos às intervenções mais relevantes a serem realizadas?

18. São utilizados parâmetros e metas com base na necessidade da população para programação das ações em saúde bucal?

22. A APS coordena os fluxos de referência e contrarreferência em saúde bucal?

25. As ações de urgência e emergência de competência da APS são programadas/planejadas para serem ofertadas na unidade?

Questões excluídas do instrumento original

$16-17,19,21,23,30-32$

Questões acrescentadas

13. As unidades de atenção primária em saúde bucal possuem infraestrutura adequada ao seu funcionamento (sala, consultório, equipamento, instrumental, material de consumo)?

14. As unidades de atenção primária em saúde bucal possuem equipe modalidade I e/ou II?

15. Existe uma definição clara das ações/procedimentos a serem desenvolvidas pela equipe de saúde bucal?

16. A atenção primária em saúde bucal realiza todas as ações/procedimentos de sua competência?

19. Existe trabalho integrado entre os diversos profissionais da equipe de APS da Unidade?

20. Os ACS participam das ações de saúde bucal?

Domínio: OS PONTOS DE ATENÇÃO À SAÚDE SECUNDÁRIOS E TERCIÁRIOS

Questões excluídas do instrumento original

34-74

Questões acrescentadas

27. Há referências ambulatoriais especializadas, de nível secundário, para estomatologia?

28. Há referências ambulatoriais especializadas, de nível secundário, para a confecção de próteses unitárias?

29. Há referências ambulatoriais especializadas, de nível secundário, para cirurgia oral menor?

30. Há referências ambulatoriais especializadas, de nível secundário, para periodontia?

31. Há referências ambulatoriais especializados, de nível secundário, para endodontia?

32. Há referências ambulatoriais especializadas, de nível secundário, para os cuidados aos usuários com necessidades especiais?

33. Há referências ambulatoriais especializadas, de nível secundário, para ortodontia?

34. Há referências ambulatoriais especializadas, de nível secundário, para implantodontia?

35. Há referências de nível terciário para tratamento de disfunção da ATM e dor orofacial?

36. Há referências hospitalares, de nível terciário, para o tratamento odontológico do usuário com necessidades especiais com analgesia/sedação/anestesia geral?

37. Há referências hospitalares de nível terciário para os cuidados aos traumatismos bucomaxilofaciais?

38. Há referências de nível terciário, para os cuidados às urgências e emergências odontológicas em regime de plantão 24 horas?

39. Há referências hospitalares, de nível terciário, para o tratamento do câncer bucal?

40. Há referências de nível terciário para tratamento das anomalias crânio-faciais?

41. Os níveis de atenção especializada em saúde bucal respeitam a atenção primária como porta de entrada e coordenadora da rede?

42. Os protocolos clínicos estabelecem claramente as relações dos serviços ambulatoriais e hospitalares com a atenção primária à saúde?

43. Existe uma definição das ações / procedimentos a serem ofertados por cada ponto de atenção especializada?

44. Existem e são utilizados protocolos clínicos relativos às intervenções mais relevantes a serem realizadas?

45. Os pontos de atenção especializada em saúde bucal realizam todos os procedimentos de sua competência? 
Figura 1 (continuação)

\begin{tabular}{|c|c|}
\hline \multicolumn{2}{|r|}{ Domínio: OS SISTEMAS DE APOIO } \\
\hline \multicolumn{2}{|r|}{ Questões excluídas do instrumento original } \\
\hline \multicolumn{2}{|r|}{$75-89$} \\
\hline \multicolumn{2}{|r|}{ Questões acrescentadas } \\
\hline 46. & O sistema de assistência farmacêutica disponibiliza os medicamentos de saúde bucal previstos no protocolo clínico? \\
\hline 47. & Existe sistema de apoio para realização de exames radiológicos em odontologia? \\
\hline 48. & Há protocolos clínicos que orientam a indicação e o fluxo dos exames radiológicos em odontologia e sua interpretação (laudo)? \\
\hline & Existe sistema de apoio para realização de exames de análise histopatológica em odontologia? \\
\hline & Há protocolos clínicos que orientam a indicação e o fluxo dos exames de análise histopatológica em odontologia e sua interpretação (laudo)? \\
\hline & Existe sistema de apoio para realização de exames de análises clínicas para a odontologia? \\
\hline 52. & Há protocolos clínicos que orientam a indicação e o fluxo dos exames de análises clínicas em odontologia e sua interpretação? \\
\hline & Existe um sistema de manutenção dos equipamentos odontológicos? \\
\hline & Existe um apoio de laboratório de prótese dentária? \\
\hline & Existe um apoio de laboratório de prótese bucomaxilofacial? \\
\hline & Existe um apoio de laboratório para confecção de aparelhos ortodônticos? \\
\hline & Existe um prontuário clínico (em papel ou eletrônico) que integra as informações epidemiológicas e gerenciais? \\
\hline & Há uma base de dados única que incorpora toda população usuária? \\
\hline & Esta base de dados única estratifica em subpopulações por riscos? \\
\hline & Existe um fluxo regular de alimentação dos procedimentos de saúde bucal no SIA/SUS e SIAB? \\
\hline \multicolumn{2}{|r|}{ Domínio: OS SISTEMAS LOGÍSTICOS } \\
\hline \multicolumn{2}{|r|}{ Questões mantidas do instrumento original } \\
\hline & O prontuário familiar eletrônico permite a emissão de receitas eletrônicas? \\
\hline & O prontuário familiar eletrônico está construído para emitir lembretes e alertas e dar feedbacks relativos a situações de saúde definidas? \\
\hline \multicolumn{2}{|r|}{ Questões adaptadas do instrumento original } \\
\hline & A equipe de atenção primária à saúde bucal agenda diretamente as intervenções eletivas na atenção ambulatorial especializada? \\
\hline & A equipe de atenção primária à saúde bucal agenda diretamente alguns exames de análises clínicas e de patologia clínica ou de imagens? \\
\hline \multicolumn{2}{|r|}{ Questões excluídas do instrumento original } \\
\hline \multicolumn{2}{|r|}{$90-94,98,100-105$} \\
\hline \multicolumn{2}{|r|}{ Questões acrescentadas } \\
\hline & Há um cartão de identificação dos usuários (Cartão SUS) com um número único para cada pessoa, articulado com a identificação da familia e do território sanitário? \\
\hline & Em relação ao prontuário clínico: inexistente 0; existente (em papel ou eletrônico) individual 1; existente (em papel) familiar 2; existente (eletrônico) familiar 3. \\
\hline & Há um prontuário familiar eletrônico único que pode ser acessado nos diferentes pontos de atenção à saúde e nos sistemas de apoio da rede? \\
\hline & $\begin{array}{l}\text { O prontuário familiar eletrônico integra as informações da atenção primária à saúde bucal, da atenção ambulatorial especializada, da atenção hospitalar, de assistência } \\
\text { farmacêutica, e os exames de análises clínicas, patológicas e de imagens? }\end{array}$ \\
\hline & Existe uma central de regulação para agendamento dos atendimentos especializados ou de urgência/emergência 24 horas em odontologia? \\
\hline & $\begin{array}{l}\text { Há um sistema de transporte sanitário que integra a APS com outros niveis de atenção com um módulo de transporte de usuários para procedimentos eletivos de saúde } \\
\text { bucal prestado com veículos adequados, com rotas pré-definidas? }\end{array}$ \\
\hline & O sistema tem um módulo de transporte de usuários para procedimentos eletivos de saúde bucal prestado com veículos adequados? \\
\hline \multicolumn{2}{|r|}{ Domínio: O SISTEMA DE GOVERNANÇA DA REDE } \\
\hline \multicolumn{2}{|r|}{ Questões adaptadas do instrumento original } \\
\hline 78. & As gerências dos pontos de atenção à saúde (da APS, do CEO, da Unidade Odontológica Hospitalar) e dos sistemas de apoio conhecem e aderem aos objetivos da rede? \\
\hline & Os incentivos financeiros estão alinhados com os objetivos da rede de saúde bucal? \\
\hline \multicolumn{2}{|r|}{ Questões excluídas do instrumento original } \\
\hline & 106-114, 116, 118-119 \\
\hline & Questões acrescentadas \\
\hline & Existe um comitê gestor composto por representantes das secretarias municipais e estadual, da atenção primária, do CEO e da atenção hospitalar? \\
\hline & Há um sistema de gestão estratégica da rede que envolva planejamento, monitoramento e avaliação? \\
\hline & Esse sistema contempla a avaliação dos usuários? \\
\hline & Existe um sistema de acreditação da rede de atenção à saúde bucal realizado por Instituição Externa vinculado à Organização Nacional de Acreditação (ONA)? \\
\hline & Há um sistema de contratualização dos prestadores de serviços pela SMS ou SES, com base em procedimentos pré-estabelecidos? \\
\hline & Esse sistema de contratualização está alinhado com os objetivos da rede e contém incentivos para o alcance desses objetivos? \\
\hline & As ClB estadual e regionais são eficazes no mecanismo de gestão da rede? \\
\hline & A PPI é utilizada no mecanismo de gestão da rede? \\
\hline
\end{tabular}

(continua) 


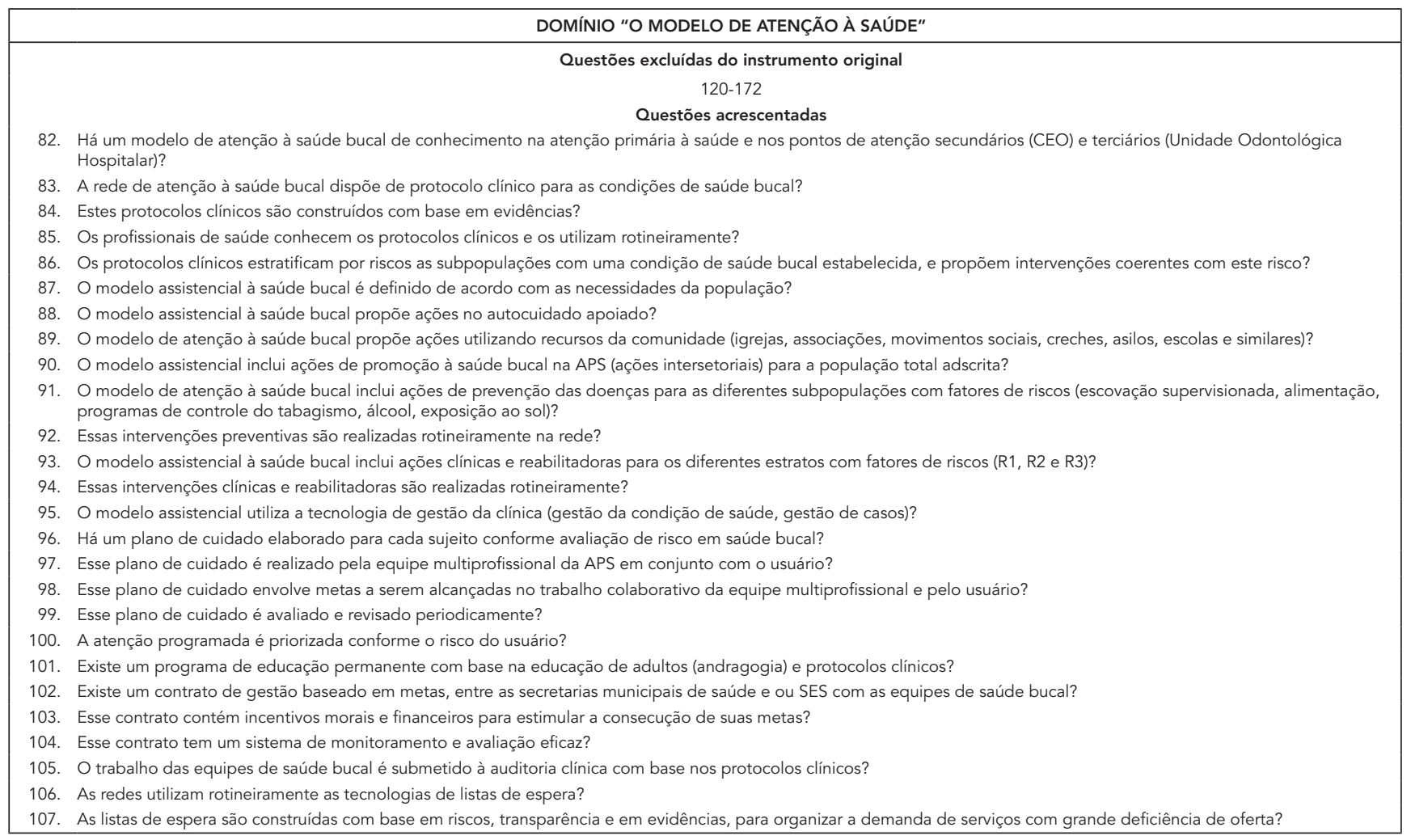

ACS: agentes comunitários de saúde; APS: atenção primária à saúde; ATM: articulação temporomandibular; CEO: centro de especialidades odontológicas; CIB: Comissão Intergestores Bipartite; PPI: Programação Pactuada e Integrada; SIAB: Sistema de Informação da Atenção Básica; SIA/SUS: Sistema de Informações Ambulatoriais do SUS.

logísticos", duas foram adaptadas, 12 excluídas e sete acrescentadas. No domínio "o sistema de governança da rede", foram adaptadas duas questões, 12 excluídas e oito acrescentadas. No domínio "o modelo de atenção à saúde", foram excluídas todas as questões do original e acrescentadas 26. Dessa forma, a versão saúde bucal apresentou um total de 107 itens, sendo menor que o original.

A versão saúde bucal seguiu a mesma estrutura do original por domínios, com convergência das questões de cada domínio e pontuação dos escores. As respostas são mensuradas em escala Likert. São quatro opções de resposta (0 - não existência do que está sendo perguntado; 1 funcionamento básico ou incipiente; 2 - funcionamento razoavelmente bom, mas insuficiente; 3 - existência em operação de forma ótima). A pontuação mínima da versão saúde bucal é 0 e a máxima 321.
Os vinte participantes da fase pré-teste tinham de 29 a 54 anos de idade, sendo $57,2 \%$ do sexo feminino. A etapa mostrou que o instrumento foi de fácil aplicação, sendo bem aceito pelos coordenadores municipais.

Após tal fase, entendeu-se a versão saúde bucal pronta para ser avaliada com relação às propriedades psicométricas.

\section{Discussão}

A importância de se ter na prática um instrumento que avalie o estágio de desenvolvimento da rede de atenção à saúde bucal reside no fato de ele auxiliar na elaboração de políticas públicas voltadas para a organização da saúde bucal. Embora a literatura disponibilize o Instrumento de Avaliação da Atenção Primária (Primary Care Assessment Tool - PCATool-Brasil) 12 que busca 
avaliar aspectos de estrutura, processo e resultados dos serviços de saúde na atenção primária à saúde, ele não mensura aspectos de estrutura, processos e resultados de serviços de saúde na rede de atenção à saúde. Além disso, o PCATool, em suas diferentes versões, é aplicado por meio de entrevistas às crianças, adultos e profissionais de saúde. Já o instrumento de diagnóstico da rede de atenção à saúde é direcionado aos gestores (coordenadores de saúde), não necessitando da presença do entrevistador durante o preenchimento.

Ao se propor a adaptação de um instrumento, há necessidade de se considerar as características diversas existentes nos grupos pesquisados 8,13,14. A equivalência conceitual e funcional entre as duas versões foi obtida por intermédio da comprovação de quatro especialistas que ressaltaram a importância dos domínios para a população a que se aplica. No entanto, para que o instrumento contemplasse seu objetivo, foram propostas modificações nos itens de verificação dos domínios.

Os itens de verificação que se aplicam tanto para o instrumento original quanto para a versão saúde bucal, foram mantidos ou adaptados. Alguns domínios apresentaram poucas modificações (domínio "população" e "atenção primária á saúde”). Alguns itens de verificação do instrumento original não representam as es- pecificidades da rede de atenção à saúde bucal, mostrando-se "forçados", ou seja, exagerados em sua proposição. Requereram, portanto, exclusão e substituição.

A equivalência operacional e de itens foi realizada durante a fase de pré-teste 10,11 . Verificouse, também, a viabilidade de se utilizar, de modo similar ao instrumento original, as instruções e os métodos de medidas.

A formação em odontologia dos coordenadores de saúde bucal foi essencial para que alguns itens do questionário não gerassem dúvidas sobre conceitos específicos da área odontológica.

A importância de se ter na prática um instrumento específico para avaliar o estágio de desenvolvimento da rede de atenção à saúde bucal reside no fato de esse instrumento ser uma ferramenta importante que auxiliará as políticas públicas voltadas às especificidades de organização dos municípios.

\section{Conclusão}

O instrumento de diagnóstico do estágio de desenvolvimento da rede de atenção à saúde bucal apresentou-se adaptado. Porém, para ser utilizado, necessita de ter as propriedades psicométricas como parte complementar do processo de avaliação.

\section{Resumen}

La aplicación del cuidado de la salud bucal en red es un proceso reciente y necesita ser evaluado. Este estudio tuvo como objetivo describir las medidas de adaptación de un instrumento para evaluar el estado de desarrollo de la red de atención de la salud bucal en el Sistema Nacional de Salud de Brasil. El instrumento original, llamado Instrumento de Prácticas de Diagnóstico para el Desarrollo de la Red de Salud, fue desarrollado por Mendes (2011). Realizamos una evaluación formal del sentido global y de referencia del instrumento original, llegando a la versión adaptada. Para ello, el instrumento fue revisado por un comité de expertos. Entender la versión adaptada se evaluó en un test previo aplicado a 20 coordinadores municipales de salud bucal de Minas Gerais, Brasil. Se encontró una buena equivalencia, elementos funcionales conceptuales, semánticos y funcionamiento entre la versión original y adaptada del instrumento. La salud bucal ha sido bien entendida, su presentación adecuada para que se realice la prueba de sus propiedades psicométricas. Esta etapa está a punto de concluir.

Estudios de Validación; Cuestionarios; Salud Bucal

\section{Colaboradores}

D. L. Leal contribuiu na concepção do projeto, coleta e análise dos dados, redação e discussão do artigo. S. M. Paiva colaborou na redação e revisão critica do artigo. M. A. F. Werneck participou na concepção do projeto, redação e discussão do artigo. A. C. B. Oliveira contribuiu na concepção do projeto, análise dos dados, redação e revisão crítica do artigo.

\section{Agradecimentos}

Agradecemos ao autor do instrumento original, Dr. Eugênio Vilaça Mendes por orientação na etapa de adaptação do instrumento para a saúde bucal. Agradecemos a equipe da Diretoria de Saúde Bucal da Secretaria Estadual de Saúde de Minas Gerais (SES/MG), que realizou a primeira versão da adaptação do instrumento. Agradecemos ao Prof. Dr. Samuel Jorge Moysés e ao Prof. Dr. Mauro Henrique Nogueira Guimarães de Abreu pelas contribuições ao instrumento adaptado. Somos gratos aos coordenadores municipais de saúde bucal que participaram do estudo. 


\section{Referências}

1. Ministério da Saúde. Redes regionalizadas de atenção à saúde: contexto, premissas, diretrizes gerais, agenda tripartite para discussão e proposta de metodologia para implementação (versão para debate). http://portal.saude.gov.br/portal/arqui vos/doc/documento_redes_versao_nov2008.pdf (acessado em 12/Mai/2013).

2. Kuschnir R, Chorny AH. Redes de atenção à saúde: contextualizando o debate. Ciênc Saúde Coletiva 2010; 15:2307-16.

3. Lima JC, Rivera FJU. Redes de conversação e coordenação de ações de saúde: estudo em um serviço móvel regional de atenção às urgências. Cad Saúde Pública 2010; 26:323-36.

4. Mendes EV. As redes de atenção à saúde. 2ạ Ed. Brasília: Organização Pan-Americana da Saúde; 2011.

5. Mendes EV. As redes de atenção à saúde. Ciênc Saúde Coletiva 2010; 15:2297-305.

6. Silva SF. Organização de redes regionalizadas e integradas de atenção à saúde: desafios do Sistema Único de Saúde (Brasil). Ciênc Saúde Coletiva 2011; 16:2753-62.

7. Herdman M, Fox-Rushby J, Badia X. "Equivalence" and the translation and adaptation of health-related quality of life questionnaires. Qual Life Res 1997; 6:237-47.

8. Herdman M, Fox-Rushby J, Badia X. A model of equivalence in the cultural adaptation of HRQoL instruments: the universalist approach. Qual Life Res 1998; 7:323-35.

9. Reichenheim ME, Moraes CL. Operacionalização de adaptação transcultural de instrumentos de aferição usados em epidemiologia. Rev Saúde Pública 2007; 41:665-73.
10. Torres CS, Paiva SM, Vale MP, Pordeus IA, RamosJorge ML, Oliveira AC, et al. Psychometric properties of the Brazilian version of the Child Perceptions Questionnaire (CPQ11-14) - short forms. Health Qual Life Outcomes 2009; 7:43.

11. Bonanato K, Pordeus IA, Compart T, Oliveira AC, Allison PJ, Paiva SM. Cross-cultural adaptation and validation of a Brazilian version of an instrument to assess impairments related to oral functioning of people with Down syndrome. Health Qual Life Outcomes 2013, 11:4.

12. Ministério da Saúde. Manual do instrumento de avaliação da atenção primária à saúde: Primary Care Assessment Tool (PCATool-Brasil). Brasília: Ministério da Saúde; 2010.

13. van Widenfelt BMV, Treffers PDA, de Beurs E, Siebelink BM, Koudijs E. Translation and cross-cultural adaptation of assessment instruments used in psychological research with children and families. Clin Child Fam Psychol Rev 2005; 8:135-47.

14. Guillemin F, Bombardier C, Beaton D. Cross-cultural adaptation of health-related quality of life measures: literature review and proposed guidelines. J Clin Epidemiol 1993; 46:1417-32.

Recebido em 27/Mai/2013

Versão final reapresentada em 22/Jan/2014

Aprovado em 07/Mar/2014 